advanced, and there was mural thrombus in the aorta that partially occluded the ostia of both renal and common iliac arteries. The liver was of normal size (weighing $1530 \mathrm{~g}$ ) with a smooth capsule and merely minor fatty change on slicing. The spleen weighed $430 \mathrm{~g}$ and was extremely soft. Both kidneys were enlarged, $180 \mathrm{~g}$ and $210 \mathrm{~g}$. The external surfaces were slightly granular and on slicing the corticomedullary boundaries were blurred. There was no obstruction.

The duodenum and small intestine were normal. The whole of the colon showed multiple small polyps but no evidence of active ulcerative colitis or of neoplasm. There was an area of old infarction on the inferior surface of the occipital lobe of the brain-for which there was no clinical counterpart. There was atheroma in the cerebral arteries and in all the other arteries examined.

Microscopically the kidneys showed no evidence of chronic glomerulonephritis but there was severe amyloidosis (fig 2) that also affected all the organs examined. The inflammatory infiltration of the bowel was confined to the lamina propria; no granulomata were found-features in favour of ulcerative colitis rather than Crohn's disease. There was amyloid deposition around some small vessels in the bowel wall. The liver contained striking amyloid deposition around the arteries and veins: so did the spleen, heart, pancreas, lung, pituitary, and thyroid.

The pathologist's diagnosis was: extensive systemic amyloidosis of secondary type; chronic ulcerative colitis in remission; severe generalised atherosclerosis with coronary artery stenosis and old cerebral infarction; and left ventricular hypertrophy.

PROFESSOR WRIGHT: Clinically the problem was whether one could tie the initial illness with the later disease. Perhaps Professor Milne could tell us if amyloidosis can pursue such a long course.
PROFESSOR M D MILNE (7): This seems unlikely when there was complete absence of it previously. Were the biopsy specimens examined by the retrospectoscope?

PROFESSOR EVANSON: I haven't been able to get hold of the original biopsy of 15 years ago. The more recent rectal biopsies did not show amyloid even on review but at necropsy in the bowel itself the infiltration with amyloid was pretty sparce. The liver biopsy from 1962 has not been reviewed retrospectively. Do you think there could have been amyloidosis at that time? If there were we must consider its aetiology because the distribution was that of secondary amyloidosis. Our interpretation was that the amyloidosis was secondary to his chronic ulcerative colitis, and that the original renal episode was something quite separate.

The conference was recorded and edited by $\mathrm{Dr}$ W F Whimster.

\section{APPOINTMENTS OF SPEAKERS}

(1) Professor J M Evanson, MB, FRCP, professor of medicine, University of Manchester, University Hospital of South Manchester, Manchester M20 8LR.

(2) Dr D Asbury, MB, FFR, consultant radiologist, University Hospital of South Manchester, Manchester M20 8LR.

(3) Professor R Wright, MD, FRCP, professor of medicine, University of Southampton, Royal South Hants Hospital, Southampton SO9 4PE.

(4) Dr J Hampson, MB, FRCP, physician, Friarage Hospital, Northallerton, N Yorks.

(5) Dr B Goldberg, MB, FFR, consultant radiologist, Edgware General Hospital, Edgware, Middlesex.

(6) Professor Sheila Sherlock, MD, FRCP, professor of medicine, Royal Free Hospital Medical School, London NW3 2QG.

(7) Professor M D Milne, MD, FRCP, professor of medicine, Westminster Hospital Medical School, London SW1P 2AR.

\title{
Predicting fetal death by measuring oestrogen:creatinine ratios on early morning samples of urine
}

\author{
L G S RAO
}

British Medical fournal, 1977, 2, 874-876

\section{Summary}

Total oestrogens were measured, on a creatinine basis, by a fully automated method in 75000 early morning specimens of urine from 5429 women. Among the 5241 pregnancies in which the oestrogen:creatinine ratio was normal there were 11 fetal deaths (0.21\%). But there were 57 fetal deaths $(30 \cdot 3 \%)$ among 188 patients with ratios below the fifth centile. When delivery took place before

Department of Steroid Biochemistry, Royal Infirmary, Glasgow G4 OSF

L G S RAO, MSC, PHD, senior biochemist (present address: Biochemistry Department, Maternity Hospital, Bellshill, Lanarkshire) the ratio decreased to below the fifth centile a live baby was born in every one of 59 pregnancies.

Apart from its effectiveness in predicting fetal death, the test has a high degree of precision and can be performed swiftly, and cheaply. For these reasons the assessment of fetoplacental function by measuring the urinary: creatinine ratio could become a convenient and inexpensive part of antenatal care that could be extended to every pregnant woman without making many further demands on obstetricians' time.

\section{Introduction}

A recent leading article in the $B M \mathcal{F}^{1}$ specified the requirements for a good fetoplacental test. The test should $(a)$ give a reliable warning of impending fetal death; $(b)$ give an assurance of the safety of the fetus when the results are normal; $(c)$ be technically simple and cheap enough to be carried out daily on all patients; 
and $(d)$ indicate the expected quality of life of the child. The leader also emphasised that the clinical value of such a test should be thoroughly assessed on a wide scale. I report here on three and a half years' experience of the routine use of a fetoplacental function test that appears to satisfy these requirements.

\section{Patients and methods}

From 1 July 1973 to 31 December 19765429 women in their last trimester of pregnancy were studied. In each case the obstetrician had requested an oestrogen assay, and over 75000 determinations of the oestrogen:creatinine ratios were performed. About $2 \mathrm{ml}$ of urine from the first morning specimen was sent to the laboratory in a plastic specimen tube without preservative.

Total oestrogens were determined by a continuous flow autoanalyser method, ${ }^{2}$ with slight modifications, using the Kober reaction directly on diluted urine followed by the extraction of the fluorescing material by the Ittrich procedure. Initially 40 samples per hour were processed, and this was later increased to 60 per hour. Creatinine was also determined on the same diluted urine specimen by an autoanalyser method using the alkaline picrate reaction. The normal range of oestrogen:creatinine ratios at 30 to 40 weeks of pregnancy was established on urine from outpatients who eventually had uncomplicated deliveries of normal-sized infants at 38 to 40 weeks' gestation. These women had no evidence of proteinuria or hypertension, had a normal weight gain, and had a good obstetric history.

The clinical details of the patients were made available to this laboratory by the obstetricians at our group of hospitals. The details came mainly from reports of monthly perinatal meetings, at which all perinatal deaths were discussed.

\section{Results}

Ratios in patients with fetal deaths-During the period of study there were 15608 deliveries and 166 fetal deaths. Only about onethird of these patients (5429) had requests for oestrogen assays, and in this group there were 68 fetal deaths. For various reasons the obstetricians took no action in any of these cases. Altogether 57 fetal deaths occurred among the 188 patients with oestrogen:creatinine ratios below the fifth centile (in 51 cases of fetal death the ratio was less than three), while 11 fetal deaths occurred among the 5241 patients with normal ratios. The 11 fetal deaths in patients with normal ratios at the time of fetal death were due to abruptio placentae in three cases, intrapartum asphyxia in six, rhesus sensitisation in one, and variable lie and postmaturity in one.

Ratios as a warning of impending fetal deaths-A detailed study of the 57 fetal deaths that occurred in women with low ratios showed that 10 fetuses had serious congenital abnormalities, which included anecephaly in four cases. Thirteen other fetal deaths occurred before 32 weeks' gestation. In the remaining 34 cases there was no obvious reasons why these fetuses should not have had a good chance of survival. In eight of these cases abnormally low oestrogen :creatinine ratios persisted until fetal death occurred, whereas in 15 normal ratios gradually decreased to abnormally low levels at the time of fetal death. This decrease took place over at least one week in all patients except in the one postmature pregnancy, in which there was a sudden drop in the ratio over a period of one day accompanied by the loss of fetal heart sounds (fig 1).

Need for frequent sequential assays-The oestrogen:creatinine ratio could not warn of fetal death in the 11 remaining cases of fetal death with a low ratio, because in eight of these the oestrogen:creatinine assay was requested after the loss of fetal heart sounds, and in three others the death occurred in one week between two assays. These three patients had abnormally low ratios at the time of fetal death, although the ratio had been normal a week before. More frequent assays, such as thrice a week, might have given a warning of impending fetal death in all these 11 cases, as they did in the 23 cases in which regular and frequent assays were carried out.

Live births after clinical intervention-In 59 patients whose oestrogen:creatinine ratios fell from normal to subnormal levels delivery after clinical intervention before the ratio decreased to below 3 resulted in a live infant in every case, including a set of twins. In many of these cases the reason for delivery was not necessarily a decrease in the ratio (fig 2).

Perinatal mortality-Among the group of 188 pregnancies with low oestrogen:creatinine ratios (less than the fifth centile), there were

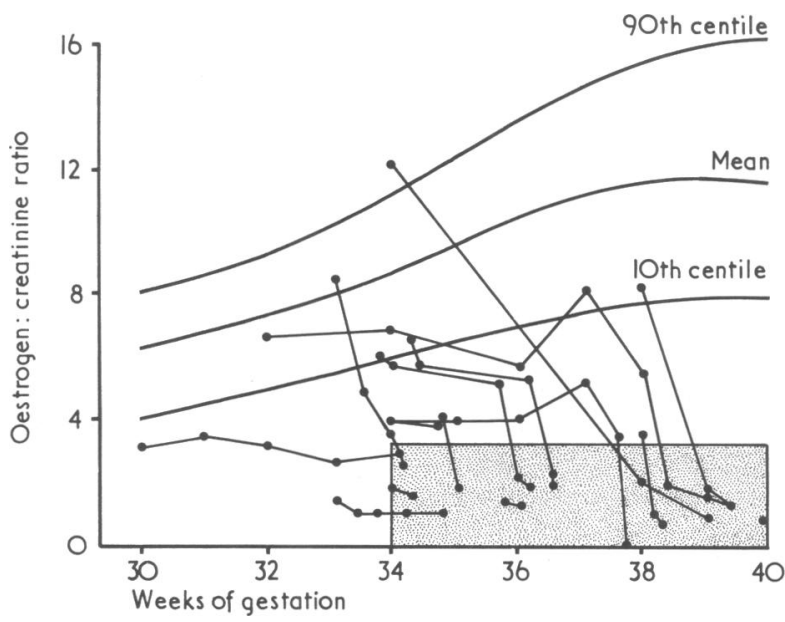

FIG 1-Oestrogen excretion in pregnancies that resulted in intrauterine death. Mean and 10th and 90th centiles are derived from ratios in normal pregnancies.

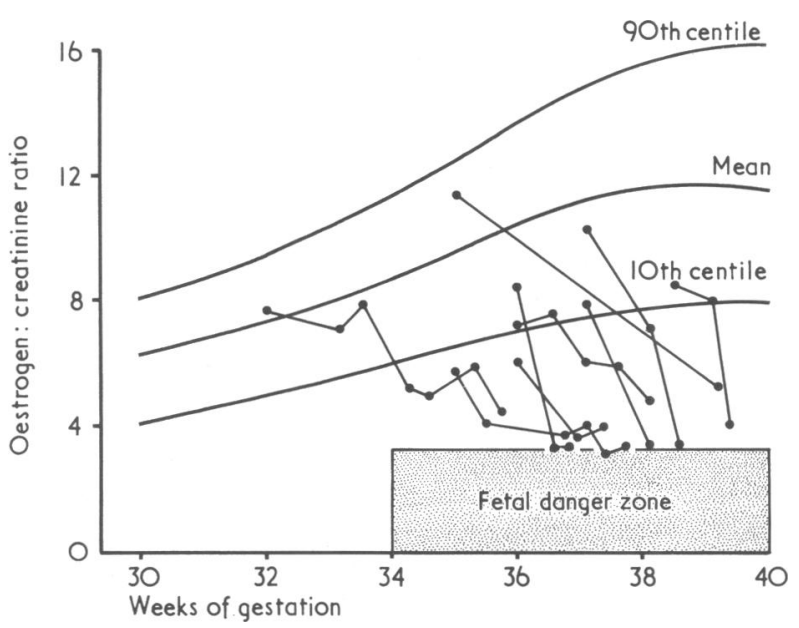

FIG 2-Live babies delivered from patients with falling levels of oestrogen. Fetal danger zone was derived retrospectively and is an area on the graph where most oestrogen:creatinine ratios were found when intrauterine death occurred.

23 neonatal deaths, which, together with the 57 fetal deaths, gave a perinatal mortality of $42.5 \%$. In the 5241 pregnancies with ratios above the fifth centile, there were 11 fetal deaths and 12 neonatal deaths. This gave a perinatal mortality of $0.44 \%$, which is about 100 times less than that among those with low ratios (see table). There was also a highly significant difference in the perinatal mortality of babies under $2500 \mathrm{~g}$ between those with low ratios $(38.7 \%$ ) and those with normal ratios $(6.0 \%)$.

Factors affecting ratio-There is a certain amount of day-to-day variability in the amount of oestrogen and creatinine measured-part of it owing to the chemical methods. The coefficient of variation within a batch of assays varied from 2 to $4 \%$ and between batches from 6 to $8 \%$. An analysis of variance showed that only the patients with preeclamptic toxaemia showed a day-to-day variation in their oestrogen: creatinine ratio that was significantly greater than that due to the

Perinatal deaths in relation to oestrogen:creatinine ratio

\begin{tabular}{|c|c|c|c|c|c|c|}
\hline \multirow{2}{*}{\multicolumn{3}{|c|}{ Oestrogen:creatinine ratio }} & \multicolumn{2}{|c|}{ Babies under $2500 \mathrm{~g}$} & \multicolumn{2}{|c|}{ All babies } \\
\hline & & & \multirow{2}{*}{$\begin{array}{c}\begin{array}{r}\text { No of } \\
\text { babies }\end{array} \\
186 \\
150 \\
547\end{array}$} & \multirow{2}{*}{$\begin{array}{l}\begin{array}{c}\text { No }\left({ }^{\prime \prime}{ }_{10}\right) \text { of } \\
\text { deaths }\end{array} \\
72(38 \cdot 7) \\
9(6 \cdot 0) \\
108(19 \cdot 7)\end{array}$} & \multirow{2}{*}{$\begin{array}{r}\begin{array}{r}\text { No of } \\
\text { babies }\end{array} \\
\begin{array}{r}188 \\
5241 \\
10179\end{array}\end{array}$} & \multirow{2}{*}{$\begin{array}{l}\begin{array}{l}\text { No }\left({ }^{0}{ }_{0}\right) \text { of } \\
\text { deaths }\end{array} \\
80(42 \cdot 6) \\
23(0 \cdot 44) \\
219(2 \cdot 2)\end{array}$} \\
\hline $\begin{array}{l}<5 \text { th centile } \\
5 \text { th centile } \\
\text { Not measured }\end{array}$ & $\begin{array}{l}\ldots \\
\cdots\end{array}$ & $\begin{array}{l}\ldots \\
\ldots\end{array}$ & & & & \\
\hline \multicolumn{3}{|c|}{ Total .. } & 883 & $189(21 \cdot 4)$ & 15608 & $322(2.06)$ \\
\hline
\end{tabular}


chemical methods. A deficiency of placental sulphatase or the administration of corticosteroids or ampicillin is known to result in the excretion of very low concentrations of oestriol, but very few patients in this study were affected by these factors, and those who were have not been included in the analysis of the results.

\section{Discussion}

This assessment of the usefulness of the oestrogen:creatinine ratio in predicting fetal death is based on a study of the ratio in many patients over three and a half years. The results show that, barring unpredictable obstetric accidents such as abruptio placentae or intrapartum asphyxia, a normal oestrogen :creatinine ratio is associated with no danger to the life of the fetus, even in the presence of maternal disease such as severe hypertension and toxaemia, and may therefore be of some reassurance to the obstetrician. On the other hand, a decrease in the ratio from normal levels to below the fifth centile is very often associated with fetal death. The risk of fetal death in this high risk zone below the fifth centile is about 100 times that in pregnancies with higher ratios.

All the data on fetal deaths were obtained in cases where the obstetricians took no action on the basis of the oestrogen: creatinine ratio. Whenever the obstetricians did intervene before the ratio decreased to below the fifth centile no fetal deaths occurred. This decrease took place over several days and allowed sufficient time for clinical intervention. Clearly, also, as has been suggested,' ${ }^{\prime}$ weekly assays are not frequent enough to predict fetal death because a normal ratio might decrease to an abnormal one within one week, resulting in fetal death. Assays should therefore be carried out at least three times a week, and preferably daily, on all pregnant women.

Sequential assays are also essential because there is no significant difference in the mean oestrogen:creatinine ratio between normal and abnormal pregnancies, and only trends are of value in assessing fetoplacental function. Daily or even thrice-weekly assays on all patients would demand very large numbers of assays, and the need for a convenient method of sample collection and laboratory assay becomes obvious. The present method can provide an efficient daily service for many patients, as up to 400 assays can be performed by one technician in a working day. In addition, this method, which does not require 24 -hour urine collection, can produce a result in less than 30 minutes after a request for an oestrogen assay.

The lack of any sharp diurnal variation ${ }^{3}{ }^{4}$ in the oestrogen: creatinine ratio makes it possible to confirm a downward trend in the ratio seen in a morning specimen by repeating the assay on a specimen collected in the afternoon of the same day. The ease of specimen collection, the large capacity of the fullyautomated method ( 60 samples per hour), and the low cost of reagents remove the practical limitations on the number of specimens that could be assayed using 24 -hour collections. Thus the oestrogen : creatinine ratio could provide inexpensive but adequate antenatal care to those patients who cannot be seen often by an obstetrician.

This test may also be valuable in giving some idea of the quality of life ${ }^{1}$ of the infant, as babies born to mothers who have low oestrogen excretion in pregnancy (in 24-hour collections) have a disproportionately higher incidence of neurological and other postnatal complications than those with normal oestrogen excretion. ${ }^{5} \mathrm{~A}$ follow-up study of all the small babies with normal or low oestrogen:creatinine ratios is in progress to test this expectation. Possibly mental retardation might be prevented by the early delivery of babies with poor fetoplacental function instead of allowing the pregnancies to continue to term. ${ }^{6}$

I am extremely grateful to Professor M C MacNaughton and Drs M M Garrey, P W Howie, A W Laughland, N MacGregor, H P McEwan, J Mowat, R Murdoch, and L Paterson for the facilities to investigate the patients under their care and for providing clinical details and useful suggestions. I should also like to thank the nursing staff of the Royal Maternity Hospital for collecting the specimens. This work would not have been possible without the encouragement and interest of Dr J K Grant, the head of this laboratory.

\section{References}

1 British Medical fournal, 1977, 1, 123.

${ }^{2}$ Hainsworth, I R, and Hall, P E, Clinica chimica acta, 1971, 35, 201.

${ }^{3}$ Dickey, R P, et al, American fournal of Obstetrics and Gynecology, 1966, 94, 591 .

4 Welshman, S G, Armstrong, M J, and Bell, J F, Clinica chimica acta 1969, 26, 339

5 Trolle, D, Beck, J, and Gaede, P, American fournal of Obstetrics and Gynecology, 1976, 126, 834.

${ }^{6}$ Rhodes, P, British Medical fournal, 1973, 1, 399.

(Accepted 26 fuly 1977)
A patient who has had two mid-trimester abortions and no other pregnancies has had a normal hysterosalpingogram. Her first miscarriage was caused by abruptio placentae, and the second was probably associated with poor placentation, causing an intrauterine death. Could there be a common factor, and what further investigations should be undertaken to exclude any predisposing cause?

Terms can confuse as much as they can clarify. This patient has evidence of failure of placental function, in the first instance acute, and in the second more chronic. It is highly likely that there is a common factor, but impossible with present knowledge to say what it might be. An association has been suggested between abruptio placentae and folic acid deficiency, operating in early pregnancy. The folic acid state might be investigated to decide if this substance might be given prophylactically, even before the next pregnancy. Generally, the results of further investigations to find predisposing causes for these intrauterine deaths are disappointing, though it is important not to overlook such obvious things as diabetes, renal disorder, syphilis, and rhesus incompatibility. More usually no overt causes will be found and the next pregnancy must be treated empirically, monitoring placental function and fetal growth closely and often, with recourse to periods of rest in bed in hospital. Depending on the woman herself and the depth of desire for a baby of her and her husband, a case can be made for her being in hospital for several months. It may or may not work but there is little else to offer, and if a third pregnancy also fails there is some merit in all concerned feeling that everything possible has been done.
It may minimise the inevitable psychological repercussions of such a personal tragedy.

I have used propanolol (Inderal) with success in a dose of $10 \mathrm{mg}$ twice a day to stop excessive sweating in a patient who had athlete's foot. Is this a recognised use for this drug, and can it be used for other skin conditions where hyperhidrosis is present?

Although Dale and Feldberg showed in 1934 that eccrine sweating was mediated through the orthosympathetic nervous system yet was parasympathetic - that is, cholinergic-in its pharmacological responsiveness, many authors have shown since $1948^{1}$ that intradermal injections of adrenergic compounds also produce eccrine sweating. This is much less evident, however, than that induced by cholinergic substances. Several patients taking propanolol have observed diminished sweating, yet others have experienced increased sweating or were not aware of any appreciable change. Diminished sweating seems commoner with oxprenolol, but this is certainly not of the same order as that induced by the established antisweating drugs such as poldine methylsulphate. The cardiac and bronchial effects of betaadrenergic blocking agents, as well as other pharmacological considerations, argue against recommending this group of drugs to diminish excessive sweating. 